\title{
Antioxidant activities and total phenolic content in germinated and non-germinated legume extracts following alkaline-acid hydrolysis
}

\begin{abstract}
This study was aimed to determine and compare Total Phenolic Content (TPC) and Antioxidant Activities (AA) in germinated and non-germinated legume [peanut (Arachis hypogeal) and soybean (Glycine max)] extracts prepared using alkaline-acid hydrolysis. Total phenolic content and antioxidant activities 1,1- diphenyl-2-picrylhydrazyl free radical (DPPH) scavenging and reducing Ferric Ion Antioxidant Potential (FRAP) in extracts were determined spectrophotometrically. Total phenolic content in non-germinated peanut and soybean were 1090.58 and $888.08 \mathrm{mg} \mathrm{GAE} / 100 \mathrm{~g}$ dry weight, respectively. After germination, total phenolic content in peanut increased to reach $1151.33 \mathrm{mg}$ GAE/100g dry weight; while soybean significantly decreased. In non-germinated peanut and soybean DPPH was 1084.99 and 507.59 عM TE/100g dry weight; and FRAP was 47966.67 and 23010.00 عM Fe2+ equivalent/100g dry weight respectively. Following germination, DPPH and FRAP were decreased in peanuts but increased in soybeans. The process of germination has resulted to the increments of antioxidant activities in soybean and total phenolic content in peanut.
\end{abstract}

Keyword: Acid-alkali hydrolysis; Antioxidant activities; Germination; Peanut; Soybean; Total phenolic content 